\title{
Relevance of intra- and inter-subband scattering on the absorption in heterostructures
}

\author{
C. Ndebeka-Bandou, ${ }^{1}$ F.Carosella, ${ }^{1}$ R. Ferreira, ${ }^{1}$ A. Wacker,${ }^{2}$ and G. Bastard ${ }^{1,3}$ \\ 1) Laboratoire Pierre Aigrain, Ecole Normale Supérieure, CNRS (UMR 8551), \\ Université P. et M.Curie, Université D. Diderot, 24 rue Lhomond F-75005 Paris, \\ France \\ 2) Mathematical Physics, Lund University, Box 118, S-22100 Lund, Sweden \\ 3) Technical University Vienna, Photonics Institute, Gusshausstrasse 27, A-1040 Vienna, \\ Austria
}

We analyze the absorption lineshape for inter-subband transitions in disordered quasi two-dimensional heterostructures by an exact calculation. The intra-subband scatterings control the central peak while the tails of the absorption line are dominated by the inter-subband scattering terms. Our numerical study quantitatively assesses the magnitude of the free carrier absorption. The accuracy of different models currently used for gain/absorption is discussed.

PACS numbers: 73.21.Ac,78.67.Pt

The demand to produce reliable terahertz and infrared devices has triggered extensive researches on intersubband transitions in semiconductor heterostructures ${ }^{1}$. A highlight is the realization and continuous improvement of Quantum Cascade Lasers (QCL) $)^{2-5}$ that emit in this frequency range. The search for improved performances of QCLs includes a better design of the layer sequence to enhance the population inversion between the two subbands involved in the lasing transition, the decrease of the non radiative paths, but also the control of the photon re-absorption by the free carriers that are present in the structure ${ }^{6-12}$. The free carrier absorption (FCA), well known from bulk structures, is transferred to a variety of intra-subband and inter-subband oblique (in two-dimensional $\vec{k}$ space) transitions in heterostructures ${ }^{13,14}$. Tailoring these (often parasitic) processes requires a thorough understanding of the nature of the optical transitions in imperfect heterostructures.

For light polarized along the growth $(z)$ axis of an ideal semiconductor heterostructure, the energy dependence of the absorption coefficient between subbands 1 and 2 is a Dirac delta function line centered at $\hbar \omega_{0}=E_{2}-E_{1}{ }^{15}$. This "atomic-like" profile stems from the fact that the electromagnetic wave coupling requires a non-vanishing $z$-dipole $\left\langle 1\left|\hat{p}_{z}\right| 2\right\rangle$, but does not affect the in-plane motion of the electrons. These motions are identical in the two subbands (i.e. plane waves, owing to the in-plane translation invariance) and their corresponding energies form identical dispersions (if we assume the same parabolic mass $\left.m^{*}\right)$. Static disorder affects the in-plane motion by introducing intra-subband $\left(V_{\mathrm{def}}^{\text {intra }}\right)$ as well as intersubband $\left(V_{\text {def }}^{\text {inter }}\right)$ couplings. Bound states usually appear below the subband edges. As a consequence, the strict selection rules leading to the Dirac lineshape are no longer expected to apply to a disordered structure. This is frequently taken into account by some effective broadening. Here we treat this feature from a fully microscopic point of view by considering the exact eigenstates of the disordered quantum well potential. Our calculations allow us to gain a complete insight into the underlying physical processes leading to the absorption in heterostructures, not only near resonance, but also far above or far below the resonance (theses regions have not deserved enough attention in the literature, even though of great technological interest). We will show that the regions far from resonance can nicely be understood by resorting to defect-induced scattering processes, and highlight the respective roles of $V_{\mathrm{def}}^{\text {intra }}$ and $V_{\mathrm{def}}^{\text {inter }}$. Finally, the complete calculation will allow us to determine the accuracy of different models currently employed to evaluate the gain/absorption of such structures.

In the presence of disorder, the heterostructure eigenstates can be written as $\Psi_{\nu}(\vec{\rho}, z)=\sum_{n} F_{\nu, n}(\vec{\rho}) \chi_{n}(z)$, with:

$$
\begin{aligned}
\frac{-\hbar^{2}}{2 m^{*}} \nabla^{2} F_{\nu, n}(\vec{\rho})+\sum_{n^{\prime}} F_{\nu, n^{\prime}}(\vec{\rho}) & V_{\operatorname{def}}^{n, n^{\prime}}(\vec{\rho}) \\
& =\left(\varepsilon_{\nu}-E_{n}\right) F_{\nu, n}(\vec{\rho})
\end{aligned}
$$

where $\vec{\rho}=(x, y), \quad F_{\nu, n}(\vec{\rho}) \quad$ is the in-plane envelope function of the $n^{t h}$ subband and $V_{\text {def }}^{n, n^{\prime}}(\vec{\rho})=$ $\left\langle\chi_{n}(z)\left|V_{\text {def }}(\vec{\rho}, z)\right| \chi_{n^{\prime}}(z)\right\rangle \cdot \chi_{n}(z)$ is the heterostructure localized part and $E_{n}$ the corresponding bound state. In the absence of defects $\nu \rightarrow(n, \vec{k}), F_{\nu}(\vec{\rho}) \rightarrow \exp (i \vec{k} \cdot \vec{\rho}) / \sqrt{S}$ and $\varepsilon_{\nu} \rightarrow E_{n}+\hbar^{2} k^{2} /\left(2 m^{*}\right)$. In the following we shall present results obtained after a numerical diagonalization of Eq. (1) within a truncated basis with the two lowest subband states $(n=1,2)$. In practice, we expand $F_{\nu, n=1,2}(\vec{\rho})$ in a plane wave basis that fulfill periodic (Born-von Karman) conditions in a $200 \times 200 \mathrm{~nm}^{2}$ box. The matrix element of the dipole coupling to light are proportional to $\left(\hat{p}_{z}\right)_{(\nu, \mu)}=\left\langle\Psi_{\nu}(\vec{r})\left|\hat{p}_{z}\right| \Psi_{\mu}(\vec{r})\right\rangle$. For defect-free structures one has the selection rules: $\left(\hat{p}_{z}\right)_{(n, \vec{k}),\left(n^{\prime}, \vec{k}^{\prime}\right)}=\left(1-\delta_{n, n^{\prime}}\right) \delta_{\vec{k}, \vec{k}^{\prime}}\left\langle\chi_{n}\left|\hat{p}_{z}\right| \chi_{n^{\prime}}\right\rangle$, reflecting the in-plane translation invariance and the non-vanishing $z$ dipole. In the following, we will be interested in the effect 
of disorder on these matrix elements.

In QCLs the interface defects are important elastic scatterers. Most features can be directly transferred to other mechanisms such as impurity ${ }^{16}$ and alloy scattering. Interface defects arise from the non ideality of the well/barrier interface at $z=z_{0}$ between two consecutive layers of the heterostructure: they can be a protrusion of the barrier material in the well (repulsive defects) or viceversa (attractive defects). For nearly ideal interfaces, the interface defects are only one monolayer thick. The scattering potential created by one disordered interface has the form

$$
V_{\text {def }}(\vec{r})=f(z) u_{\text {def }}(\vec{\rho})
$$

showing a separation on the dependence on the $z$ and $\vec{\rho}$ variables. $u_{\mathrm{def}}(\vec{\rho})$ is the superposition of localized functions centered at the randomly placed interface defects on the $(x, y)$ plane $^{14}$. For a barrier/well interface at $z=z_{0}$ and for a repulsive defect $f^{\mathrm{rep}}(z)=V_{b} \Theta(z-$ $\left.z_{0}\right) \Theta\left(z_{0}+h-z\right)$ while for an attractive defect $f^{\text {att }}(z)=$ $-V_{b} \Theta\left(z_{0}-z\right) \Theta\left(z-z_{0}+h\right)$, where $V_{b}$ is the barrier height, $h$ is the thickness of one monolayer, $\Theta(z)$ is the Heaviside function.

Here we consider a $9 / 2 / 3 \mathrm{~nm}$ GaAs $/ \mathrm{Ga}_{0.75} \mathrm{Al}_{0.25} \mathrm{As}$ double quantum well (DQW) structure with Gaussian interface defects ${ }^{14}$ placed on the two inner interfaces, with a characteristic in-plane extension of $3.6 \mathrm{~nm}$ and a fractional coverage of the surface $N_{\text {def }} \pi \sigma^{2} / S=30 \%$. Fig. 1 shows the in-plane probability distribution for two states with energies respectively equal to $E_{1}+2 \mathrm{meV}$ and $E_{2}+2 \mathrm{meV}$. The extra energies $+2 \mathrm{meV}$ are larger (respectively smaller) than the typical effective in-plane potential depths in the $E_{1}$ and $E_{2}$ subbands ( $0.4 \mathrm{meV}$ and 6 meV respectively). Hence, these two in-plane electronic distributions look very different: the quasi $E_{1}$ state is extended in the $(x, y)$ plane and approximately given by the wave-function $\Psi(x, y) \propto \cos \left(k_{0} x+\varphi_{x}\right) \cos \left(k_{0} y+\varphi_{y}\right)$, where $\varphi_{x / y}$ are phases and $k_{0}=4 \pi / 200 \mathrm{~nm}$. Its kinetic energy $\hbar^{2} k_{0}^{2} / 2 m^{*} \approx 2 \mathrm{meV}$ is significantly larger than the characteristic potential depth. In contrast, the "mostly $E_{2}$ state" is fairly localized by the interface defects. In Fig. 2 we show the matrix $\left|\left\langle\Psi_{\nu}(\vec{r})\left|\hat{p}_{z}\right| \Psi_{\mu}(\vec{r})\right\rangle\right|^{2}$ for our calculated eigenstates of the disordered heterostructure. The figure clearly displays two blurred straight regions around $\left|\varepsilon_{\mu}-\varepsilon_{\nu}\right| \approx \hbar \omega_{0}=73.8 \mathrm{meV}$, corresponding to the subband spacing in this sample. If there where no disorder, there would be no blurring since (see above) a single final state would match any given initial state. The fact that the matrix element is almost zero if the energy difference between the true states differs strongly from the intersubband spacing corresponds well with the conventional broadening picture.

In this context it has to be noted that the blurring is strongly reduced, if the wave functions $\chi_{n}(z)$ for the subbands $n=1$ and $n=2$ are of the same magnitude at each interface. Neglecting $V_{\text {def }}^{\text {inter }}$ this provides identical (or very similar) in-plane wave functions for both subbands, which provide strong selection rules for the $p_{z^{-}}$

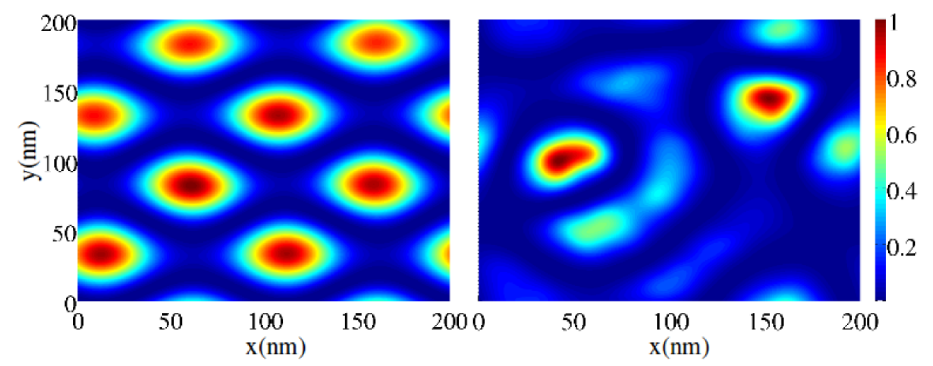

FIG. 1. Color plot of the normalized in-plane probability distribution for two states with energies equal to $E_{1}+2 \mathrm{meV}$ (left panel) and $E_{2}+2 \mathrm{meV}$ (right panel). The quasi $E_{1}$ state is extended in the $(x, y)$ plane while the quasi $E_{2}$ state is fairly localized by the interface defects.

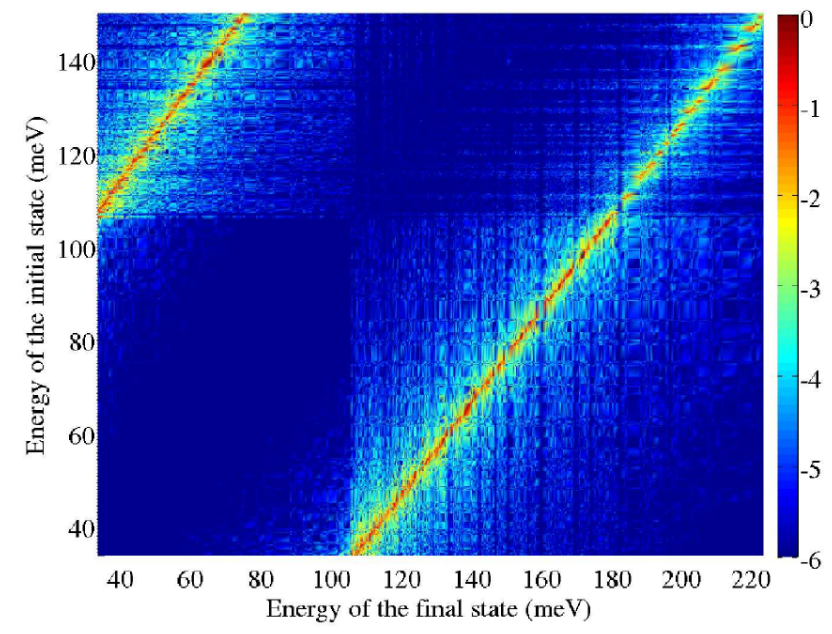

FIG. 2. Color plot of the decimal logarithm of the normalized squared modulus of the optical matrix elements for the ensemble of transitions $\varepsilon_{\nu}-\varepsilon_{\mu}$ in a disordered $9 / 2 / 3 \mathrm{~nm}$ GaAs $/ \mathrm{Ga}_{0.75} \mathrm{Al}_{0.25} \mathrm{As}$ double quantum well. The disorder is due to interface defects randomly distributed on the two inner interfaces of the structure.

matrix elements. Similar selection rules appear in strong magnetic fields ${ }^{17}$. In our case, the wave functions differ essentially at the interfaces, as the excited state penetrates deeper into the barrier, so that this effect is only of very minor relevance.

Assuming an ideal overlap between the photon modes with the angular frequency $\omega$ and the DQW structure, the absorption coefficient (in SI units) is given by :

$$
\begin{aligned}
\alpha(\omega)=\frac{2 \pi e^{2}}{m^{* 2} \omega \varepsilon_{0} c n L_{z} S} \sum_{\nu, \mu}\left(f_{\nu}\right. & \left.-f_{\mu}\right)\left|\left\langle\Psi_{\nu}\left|p_{z}\right| \Psi_{\mu}\right\rangle\right|^{2} \\
& \times \delta\left(\varepsilon_{\mu}-\varepsilon_{\nu}-\hbar \omega\right)
\end{aligned}
$$

where the thickness of one period $L_{z}$ is equal to $19.6 \mathrm{~nm}$, $n$ the refractive index and $f_{\nu}$ the occupation of the state $|\nu\rangle$ with energy $\varepsilon_{\nu}$. We show in Fig. 3 (red full line) the absorption coefficient of the DQW structure based on the matrix elements of Fig. 2 (in all absorption calculations, the delta of energy conservation was replaced by a 


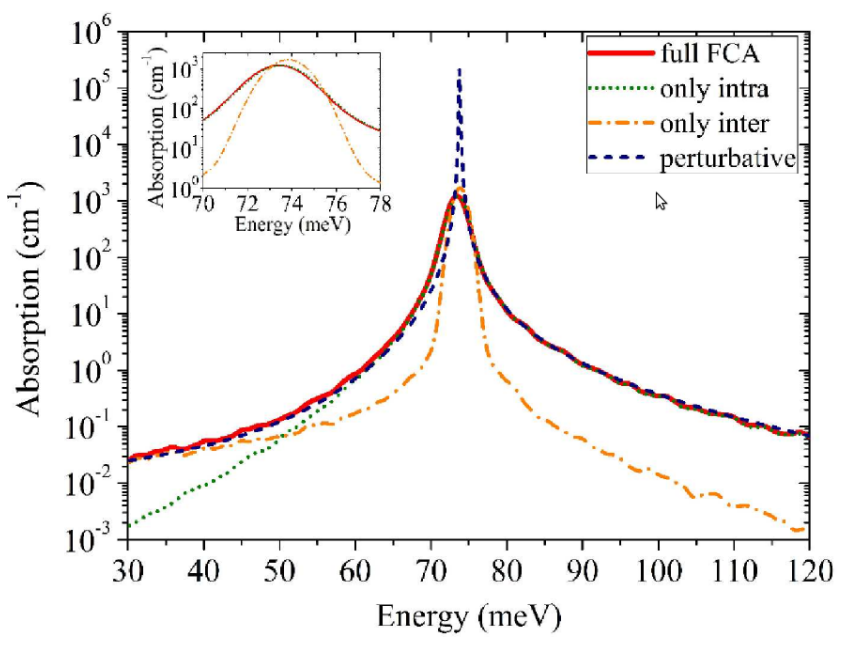

FIG. 3. Absorption spectrum for the $E_{1}-E_{2}$ transition calculated by: fully numerical diagonalization (red full line); taking into account either only $V_{\text {def }}^{\text {intra }}$ (green dotted line) or only $V_{\text {def }}^{\text {inter }}$ (orange dashed-dotted line); and by expanding the electron wavefunction to the first order in both $V_{\mathrm{def}}^{\text {intra }}$ and $V_{\mathrm{def}}^{\text {inter }}$ (see Ref. 14) (blue dashed line). Inset: Zoom of the absorption spectrum in (a) around $\hbar \omega_{0} . T=100 \mathrm{~K}$.

Gaussian with a FWHM=1.88 meV). In this absorption spectrum, the contributions of $V_{\mathrm{def}}^{\text {intra }}$ and $V_{\mathrm{def}}^{\text {inter }}$ are taken at all orders and are fully admixed. In order to highlight the physics underlying the assisted light absorption and disentangle the various contributions to its spectrum, we discuss in the following the results obtained within different approximations and/or models. We also show in Fig. 3 the calculated absorptions when only $V_{\text {def }}^{\text {intra }}$ (green dotted line) or only $V_{\text {def }}^{\text {inter }}$ (orange dashed-dotted line) are retained in the scattering matrix. We see that the low energy (the high energy) FCA is dominated by the $V_{\text {def }}^{\text {inter }}\left(V_{\text {def }}^{\text {intra }}\right)$ couplings. Indeed, the initial and final states involved in the non-resonant absorption displays a dominant $E_{1}$ or $E_{2}$ subband character. Thus, since the dipole coupling only triggers inter-subband events and starting from an initial state in $E_{1}$, an additional intersubband scattering is needed for the low energy intra- $E_{1}$ oblique transition. Conversely, the high-energy absorption tail involves essentially $E_{1}$-to- $E_{2}$ processes and thus rely mostly on $V_{\text {def }}^{\text {intra }}$. It is worth pointing out that the near-resonant absorption (see Fig. 3) is accurately accounted for by the only-intra approximation, whereas the only-inter one fails in predicting both the intensity and the absorption profile. Finally, we also show in Fig.3 the perturbative estimates of the FCA obtained by expanding the electron wavefunction to the first order in $V_{\text {def }}{ }^{14}$ (dashed blue line). We see that the perturbative estimate provides an excellent rendering of the shape and strength of the actual absorption when it is justified, i. e. not too close to the resonance.

An important issue is to understand how the results of the exact diagonalization compare to correlation function approaches, essentially based on Green's function meth- ods. To this end, we show in Fig. 4 the absorption coefficient for the $E_{1}-E_{2}$ transition of the DQW structure described above, calculated with two different correlation function (CRF) approaches, and we compare it with the one calculated by exact diagonalization. In $\mathrm{CRF}_{1}$ the Keldysh Green's function formalism was applied using the implementation of Refs. 18 and 19. In order to make a quantitatively meaningful comparison among the various calculations, we numerically extracted the correlation length and the average defect depth from the randomly generated interface defects used in the exact calculation without the use of any fitting parameters. We see that $\mathrm{CRF}_{1}$ gives a good description of the low $\omega$ behavior, as the inclusion of the nondiagonal self-energies ${ }^{18}$ fully covers the inter-subband terms addressed above. At large energy, $\mathrm{CRF}_{1}$ overestimates the absorption; a feature that is related to the use of $k$-independent scattering matrix elements in order to simplify this numerics. This assumption of an effective delta- potential scattering potential overestimates the scattering for large wave-vector transfer, which is crucial for the tails. In $\mathrm{CRF}_{2}$, we use Unuma et $a l .{ }^{20}$ formalism for the intersubband absorption coefficient that follows Ando's approach ${ }^{21}$. To draw $\mathrm{CRF}_{2}$ we converted Unuma et al.'s real part of the conductivity into an absorption coefficient following ${ }^{14}$ and we used the interface disorder potential described above. It is clear that $\mathrm{CRF}_{2}$ poorly describes the $\mathrm{FCA}$ far from resonance. This is most probably due to the fact that, contrarily to $\mathrm{CRF}_{1}$, the off-diagonal components of the Green's function are neglected, whereas the diagonal ones are evaluated within the self-consistent Born approximation. For the peak, the $\mathrm{CRF}_{1}$ and $\mathrm{CRF}_{2}$ provide similar results with a full width at half maximum of $1.8 \mathrm{meV}$ and $1.6 \mathrm{meV}$, respectively, without using any adjustable parameters. This is also in good agreement with the value of $2.5 \mathrm{meV}$ for the exact calculation (see Fig. 4). Note that the actual width of the FCA model results from a convolution between the intrinsic broadening effects (caused by scattering in an infinite sample) and the numerical broadening that we have to input in the numeric to account for the finite size of the sample in the simulation. A quantitative analysis of inter-subband linewidth should include additional scattering mechanisms (impurities, phonons) and is beyond the scope of this paper.

In conclusion, we have computed numerically the lineshape of inter-subband transitions in heterostructures with interface disorder. We have found that only few final states are optically connected to a given initial state despite disorder, which reflects the common picture of a broadened transition. While intrasubband scattering dominates at the resonance peak and at higher photon energies, intersubband scattering dominates the low energy tail of the absorption spectrum. We have compared the outputs of several models to compute absorption to the fully numerical results, in particular their low $\omega$ behaviors. The perturbative estimate for $\mathrm{FCA}^{14}$ works very well for the absorption tails. We find also that Unuma et al.'s model ${ }^{20}$ gives a poor description of the 


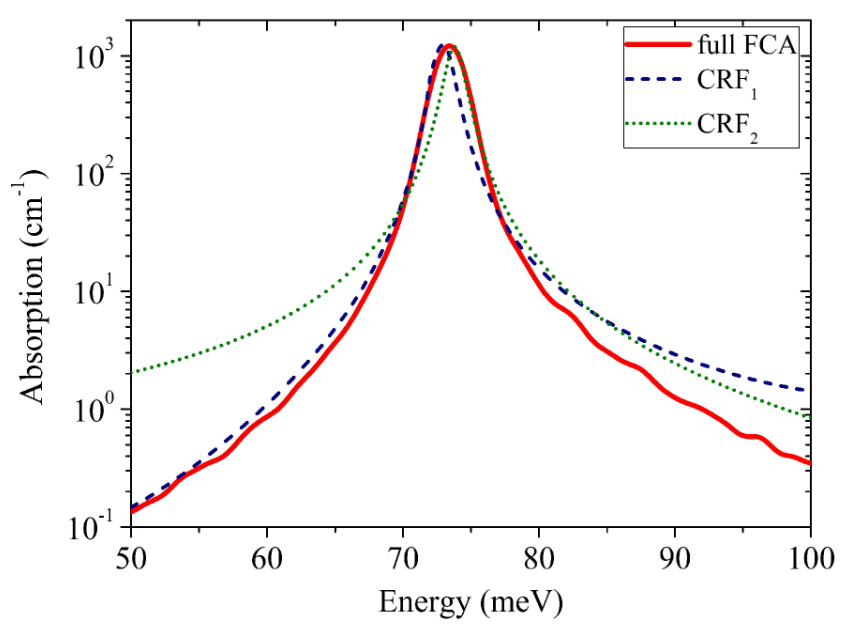

FIG. 4. Absorption spectrum for the $E_{1}-E_{2}$ transition calculated by three different models: exact diagonalization (red full line), Keldysh Green's function formalism, $\mathrm{CRF}_{1}$ (blue dashed line), Unumas model, $\mathrm{CRF}_{2}$ (green dotted line). $T=100 \mathrm{~K}$.

non-resonant FCA tails, while the Keldysh Green's function formalism ${ }^{18}$ with full nondiagonal self-energies fits the numerical results nicely. A clear picture of the intersubband and intra-subband transitions now emerges from this study and emphasizes the need for a proper account of the perturbation of the carrier wavefunctions by defects and not only of their energies.

\section{ACKNOWLEDGMENTS}

We thank E. Dupont, K. Unterrainer and G. Strasser for valuable discussions. A. Wacker thanks the Swedish Research Council (VR) for financial support.
${ }^{1}$ M. Helm, in Intersubband Transitions in Quantum wells, Semiconductors and Semimetals, Vol. 62, edited by H. Liu and F. Capasso (Elsevier, 1999) pp. 1-99

${ }^{2}$ J. Faist, F. Capasso, D. L. Sivco, A. Hutchinson, C. Sirtori, and A. Y. Cho, Science 243, 553 (1994)

${ }^{3}$ C. Walther, C. Fisher, G. Scalari, R. Terazzi, N. Hoyler, and J. Faist, Appl. Phys. Lett. 91, 131122 (2007)

${ }^{4}$ B. S. Williams, Nature Photonics 1, 525 (2007)

${ }^{5}$ S. Kumar, C. W. I. Chang, Q. Hu, and J. L. Reno, Appl. Phys. Lett. 95, 141110 (2009)

${ }^{6}$ H. Willenberg, G. H. Döhler, and J. Faist, Phys. Rev. B 67, 085315 (2003)

${ }^{7}$ H. C. Liu, M. Wätcher, D. Ban, Z. R. Wasilewski, M. Buchanan, G. C. Aers, J. C. Cao, S. L. Feng, B. S. Williams, and Q. Hu, Appl. Phys. Lett. 87, 141102 (2005)

${ }^{8}$ I. Vurgaftam and J. R. Meyer, J. Appl. Phys. 99, 123108 (2006)

${ }^{9}$ L. Ajili, G. Scalari, M. Giovannini, N. Hoyler, and J. Faist, J. Appl. Phys. 100, 043102 (2006)

${ }^{10}$ J. Faist, Appl. Phys. Lett. 90, 253512 (2007)

${ }^{11}$ J. B. Khurgin, Appl. Phys. Lett. 93, 091104 (2008)

${ }^{12}$ E. Benveniste, S. Laurent, A. Vasanelli, C. Manquest, C. Sirtori, F. Teulon, M. Carras, and X. Marcadet, Appl. Phys. Lett. 94, 081110 (2009)

${ }^{13}$ A. Wacker, G. Bastard, F. Carosella, R. Ferreira, and E. Dupont, Phys. Rev. B 84, 205319 (2011)

${ }^{14}$ F. Carosella, C. Ndebeka-Bandou, R. Ferreira, E. Dupont, K. Unterrainer, G. Strasser, A. Wacker, and G. Bastard, Phys. Rev. B 85, 085310 (2012)

${ }^{15} \mathrm{G}$. Bastard, Wave Mechanics Applied to Semiconductor Heterostructures (EDP Science, Paris, 1996)

${ }^{16}$ D. Stehr, C. Metzner, M. Helm, T. Roch, and G. Strasser, Phys. Rev. Lett. 95, 257401 (2005)

${ }^{17}$ F. Carosella, R. Ferreira, G. Strasser, K. Unterrainer, and G. Bastard, Phys. Rev. B 82, 033307 (2010)

${ }^{18}$ F. Banit, S.-C. Lee, A. Knorr, and A. Wacker, Appl. Phys. Lett. 86, 41108 (2005)

${ }^{19}$ A. Wacker, R. Nelander, and C. Weber, Proc. SPIE 7230, 72301A (2009)

${ }^{20}$ T. Unuma, M. Yoshita, T. Noda, H. Sakaki, and H. Akiyama, J. Appl. Phys. 93, 1586 (2003)

${ }^{21}$ T. Ando, A. Fowler, and F. Stern, Rev. Mod. Phys. 54, 437 (1982) 